\title{
Polymicrobial abscess following ovariectomy in a mouse
}

\author{
Victoria E. Eaton ${ }^{1,2}$, Samuel Pettit ${ }^{1}$, Andrew Elkinson ${ }^{1}$, Karen L. Houseknecht ${ }^{1}$, Tamara E. King ${ }^{1,2}$ and \\ Meghan May ${ }^{1 *}(1)$
}

\begin{abstract}
Background: Ovariectomy is a common procedure in laboratory rodents used to create a post-menopausal state. Complications including post-surgical abscess are rarely reported, but merit consideration for the health and safety of experimental animals.

Case presentation: A female C57/black6 mouse was ovariectomized as part of a cohort study. At Day 14 postsurgery, she developed a visible swelling on the right side, which 7 days later increased in size over $24 \mathrm{~h}$, leading to euthanasia of the animal. Gross pathology was consistent with abscess. A core of necrotic tissue was present in the uterine horn. Abscess fluid and affected tissue were collected for Gram stain and bacteriological culture. The abscess core and fluid yielded three distinct types of bacterial colonies identified by 165 ribosomal RNA sequencing as Streptococcus acidominimus, Pasteurella caecimuris, and a novel species in the genus Gemella.

Conclusions: This is the first report of polymicrobial abscess in a rodent as a complication of ovariectomy, and the first description of a novel Gemella species for which we have proposed the epithet Gemella muriseptica. This presentation represents a potential complication of ovariectomy in laboratory animals.
\end{abstract}

Keywords: Ovariectomy, Abscess, Polymicrobial abscess, Mouse, Streptococcus acidominimus, Pasteurella caecimuris, Gemella, Gemella muriseptica

\section{Background}

Ovariectomy (OVX) is a commonly-used model of postmenopausal age in rodent models of osteoporosis and bone fragility, impacts of menopause on dopaminergic neurons and other forms of neurodegeration, and adulthood cell differentiation [1-4].

Infection and specifically abscess is known as a potential complication of survival surgeries in laboratory mice [5]; however, information on the frequency and most common causative agents is surprisingly sparse. The most common agents of spontaneously occurring skin abscess in pet rodents are Staphylococcus aureus, Streptococcus pyogenes, and Pasteurella pneumotropica; however, (naturally occurring) intra-abdominal abscess is rarely discussed [6]. As prey animals, mice instinctively do not display overt signs of distress during moderate pain or sickness as a means of avoiding predation

\footnotetext{
* Correspondence: mmay3@une.edu

'Department of Biomedical Sciences, University of New England, 11 Hills

Beach Road, Biddeford, ME 04005, USA

Full list of author information is available at the end of the article
}

[7]. Post-surgical infection can be difficult to detect in rodents lacking overt sickness behaviors, perhaps leading to an underestimation of its rate of occurrence. Here we report a case of polymicrobial abscess following OVX in a mouse exhibiting normal behavior and no overt signs of distress.

\section{Case presentation History}

A female C57/black6 mouse (Charles River Laboratories, Wilmington, MA) underwent OVX as previously described [8] as part of an 8 animal cohort. All surgical tools and silk sutures were sterilized prior to use, and the surgical field was disinfected with chlorhexidine followed by betadine. The surgical site on the mouse was sterilized with $70 \%$ ethanol followed by betadine prior to incision. All OVX procedures for the cohort proceeded unremarkably.

(c) The Author(s). 2019 Open Access This article is distributed under the terms of the Creative Commons Attribution 4.0 International License (http://creativecommons.org/licenses/by/4.0/), which permits unrestricted use, distribution, and reproduction in any medium, provided you give appropriate credit to the original author(s) and the source, provide a link to the Creative Commons license, and indicate if changes were made. The Creative Commons Public Domain Dedication waiver (http://creativecommons.org/publicdomain/zero/1.0/) applies to the data made available in this article, unless otherwise stated. 


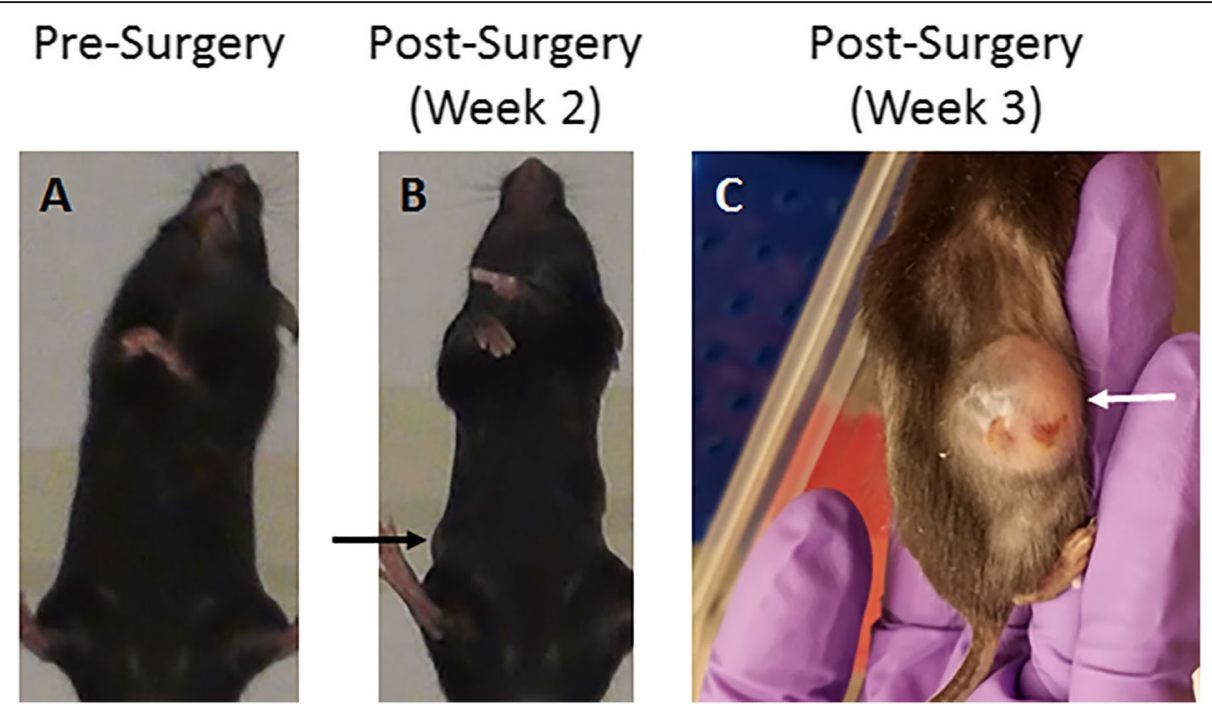

Fig. 1 Post-Surgical Evaluation. The case study subject mouse had no apparent swelling prior to OVX (panel a), and slight swelling became apparent 2 weeks post-OVX (panel $\mathbf{b}$; black arrow). The abscess increased substantially in size overnight during Week 3 (panel $\mathbf{c}$; white arrow) leading to the immediate euthanasia of the animal

\section{Clinical findings}

Two weeks following OVX, a slight swelling became apparent on the right side of the abdomen (Fig. 1b). Close monitoring indicated minimal growth of the swelling and no apparent impacts on behavior, weight, or food intake. No other signs of distress such as porphyrin rings around the eyes or rough hair coat or hair loss were observed by the experimenters or care staff. On days 20 to 21 post-OVX, the swelling increased substantially in size within $24 \mathrm{~h}$ (Fig. 1c). Though the subject was still showing no signs of distress or sickness behavior, she was euthanized because overt swelling/abscess is a humane endpoint criterion for rodents [9]. Euthanasia was carried out by pumping $100 \% \mathrm{CO}_{2}$ into a sealed chamber at $25 \%$ chamber volume per minute, per approved IACUC protocol. No other animals in the cohort developed abscesses.

Postmortem evaluation was consistent with postsurgical abscess. Purulent exudate was apparent into the peritoneal cavity, and a small, walled-off abscess core was located within the uterine horn. Exudate from the peritoneum and the abscess core were subjected to bacteriological culture for typical and atypical organisms using blood agar (Remel via Thermo Fisher Scientific, Waltham, MA) and SP-4 agar supplemented with 10\% glucose and $15 \%$ fetal bovine serum [10]. Both specimens yielded growth of two colony types on blood agar and three on SP-4 (Fig. 2a).

\section{Diagnostic procedures}

Colonies were propagated in axenic broth culture (SP-4 broth) for preservation, Gram stain, and molecular identification. Genomic DNA was extracted using EasyDNA reagents (Invitrogen via Fisher Scientific, Waltham, MA), and the 16S rRNA gene was amplified as previously described using the following primer sequences: 5 AGA GTTTGATCCTGGCTCAG $3 ; 5$ ACGGCTACCT TGTTACGACTT 3 [11]. Amplicons were subjected to Sanger sequencing using standard di-deoxy labelling methods (GeneWiz, Inc. Piscataway, NJ). Bacterial identification was made via BLAST analysis [12].

\section{Bacteriological findings}

The first colony type could only be cultivated on SP-4 agar and consisted of Gram positive diplococci (Fig. 2b). Ribosomal RNA sequencing identified this organism as a member of the genus Gemella, but the highest identity (Gemella palaticanis, GenBank accession JN713253) was only $95 \%$. Threshold for ribosomal identity between bacterial species is widely considered $97.5 \%$ [13]; therefore, this isolate likely represents a novel, previously undescribed species. The second colony type organisms were Gram positive cocci that formed long chains (Fig. 2c) and were identified as Streptococcus acidominimus (98\% identity with $S$. acidominimus strain LMG 17755, GenBank accession NR_104972). The third colony type organisms were Gram negative rods (Fig. 2d) and identified as Pasteurella caecimuris (99\% identity with $P$. caecimuris strain AA-424-CC-1, GenBank accession NR_144618).

\section{Gemella Species Characterization}

An unrooted, neighbor-joining tree based on $16 \mathrm{~S}$ ribosomal RNA sequence from several Gemella species was generated using MEGA version 7.0 [14](Fig. 3). Input 

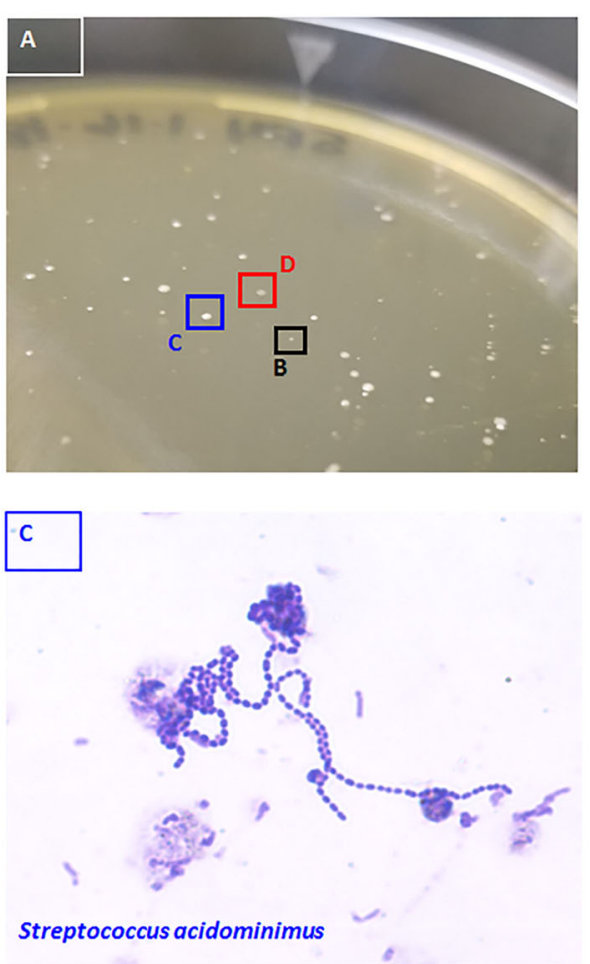
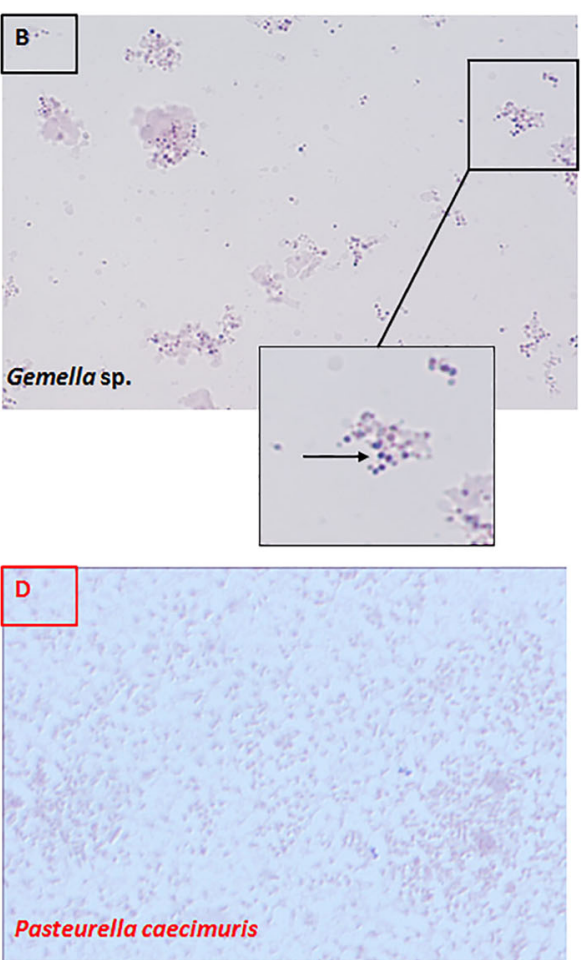

Fig. 2 Bacteriological Identification. Three colony types were recovered on SP-4 agar (panel a). Each type was identified as a distinct organism, as shown by Gram stain in panels (b-d). S. acidominimus (c) and P. caecimuris (d) showed classic morphology and arrangements of their respective genera. The novel Gemella species ('G. muriseptica') were Gram positive diplococci (Panel b inset, arrow), and bacteria tended to aggregate serum proteins present in the SP-4 broth

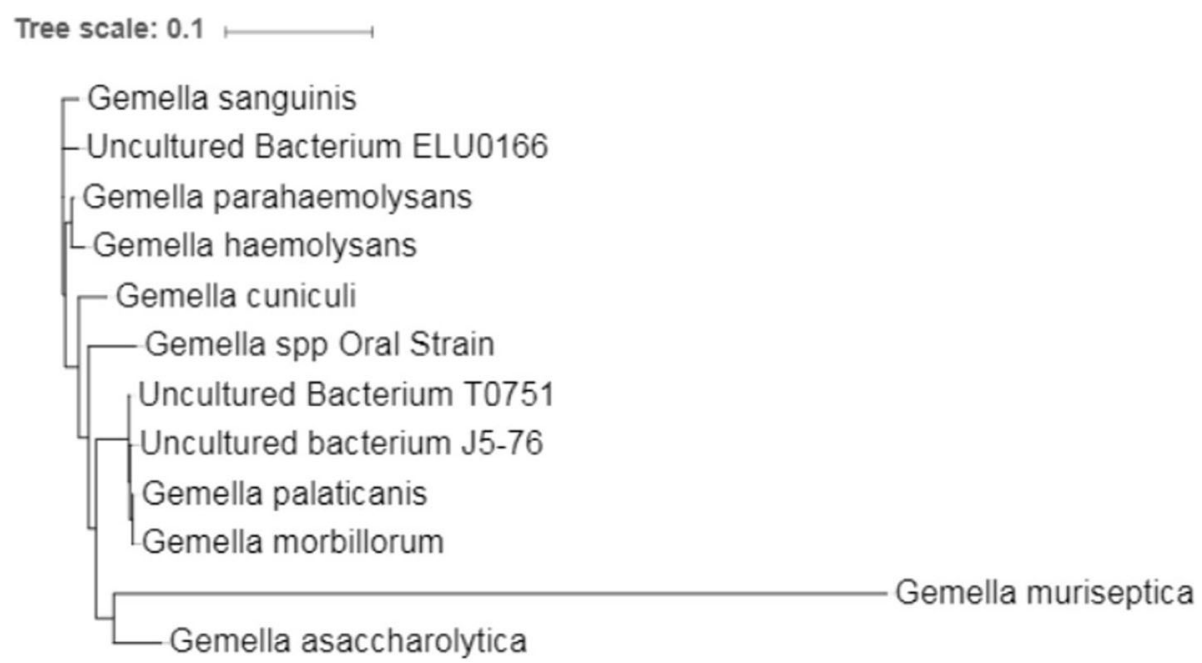

Fig. 3 Phylogeny of Gemella muriseptica. An unrooted neighbor-joining tree was generated based on 16S rRNA gene sequences from several species in the genus Gemella, as well as uncultivated sequences obtained from mammalian metagenomic studies. G. muriseptica is a divergent member of the genus, and is closest branching relative is Gemella asaccharolytica 
sequences were obtained from GenBank, and included those from G. palaticanis, Gemella sanguinis, Gemella parahaemolysans, Gemella haemolysans, Gemella cuniculi, Gemella morbillorum, Gemella asaccharolytica, Gemella species Oral Strain, and three uncultivated species from metagenomics studies ('uncultivated bacterium' ELU0166, T0751, and J5-76). Phenotypic characterizations were made using the BBL Crystal Identification reagents (Becton, Dickinson and Company, Franklin Lakes, NJ) (Gram Positive and Gram negative non-fermenter reagents), 5\% sheep's blood agar, SP-4 agar supplemented with $10 \%$ lactose, and phase-contrast microscopy. Phenotypic traits are shown in Table 1 , and are consistent with the determination that this isolate represents a novel species.

Gemella muriseptica, species nova. Gemella muriseptica (mur.i.sep'ti.ca. L. neut; L. fem. Muris- pertaining to mice; septica- indicating infection. Muriseptica- from an infected mouse). Cells are small, Gram positive cocci appearing in pairs or tetrads. Catalase- and oxidasenegative. Colonies grow on sheep's blood agar within $72 \mathrm{~h}$ and are non-hemolytic. Sucrose, arabinose, maltose, glycogen, mannitol, galactose, $\mathrm{N}$-acetyl-glucosamine, trehalose, mannose, melibiose, rhamnose, sorbitol, adonitol, and inositol are used as carbon sources. The type strain is $\mathrm{T} 3 \mathrm{C}^{\mathrm{T}}$, isolated from a murine abscess.

\section{Discussion and conclusions}

Here we report a case of postsurgical polymicrobial abscess in a laboratory animal. The biphasic nature of disease progression suggests that a slow-growing abscess caused by one organism was superinfected by the second and third, although biphasic abscess has been reported during G. morbillorum monoinfection $[15,16]$. Because all three bacterial isolates were cultivated from both specimens, it is not possible to determine the order of infection.

This case features the report of a novel Gemella species. While no members of the genus have been detected in rodents previously, metagenomic studies show that they are common inhabitants of the oral cavity and intestinal tracts of other mammals and can be detected in wastewater [17-20]. In addition, they are known to be rare causes of endogenous infection (including abscess) in humans [21-26]. This isolate is phenotypically distinct from other species, while retaining the traits required for inclusion within this genus (i.e., positive Gram stain, coccus shape, lack of motility, and absence of catalase and oxidase production) [27]. We propose the specific epithet Gemella muriseptica, species nova, for this isolate. This report also features the first isolate of $S$. acidominimus grown in axenic culture from a rodent. However, it is clear from previous metagenomics analyses that this organism is a common inhabitant of the murine microbiota (GenBank accession numbers FJ893080, KF658228, KJ910367), ( [28]), and that it has the potential for abscess formation in mice (GenBank accession number JF912770). Detection of $P$. caecimuris is not necessarily surprising, given its presence in the gut microbiome of healthy mice [29] and the ascription of $P$. pneumotropica, which holds some taxonomic confusion with $P$. caecimuris [30], to rodent abscess.

The relative rarity of reports describing post-surgical abscess in mice is potentially very consequential. Moderate levels of pain and distress can be notoriously difficult to detect in rodents because readily revealing injury or

Table 1 Gemella muriseptica Phenotypic Traits

\begin{tabular}{lllll}
\hline Carbohydrate Utilization & Rxn & Amino Acid Degradation & Rxn & Additional Phenotypes \\
\hline Sucrose & + & Proline & - & $\beta$-glucosidase \\
Arabinose & + & Arginine & - & a-glucosidase \\
Maltose & + & Methionine & + & Cellobiosidase \\
Dextrin & + & Phenylalanine & + & $\beta$-glucoronidase \\
Mannitol & + & Valine & - & Phosphatase \\
Galactose & + & Tryptophan & - & B-galactosidase \\
N-acetylglucosamine & + & Glutamic acid & Urease & - \\
Trehalose & + & Ornithine & + & Catalase \\
Mannose & + & Lysine & - & Oxidase \\
Melibiose & + & Glycine & - & Esculin hydrolysis \\
Rhamnose & & & Hemolysis \\
Sorbitol & + & & Citrate utilization \\
Adonitol & + & & N-acetylglucosaminidase \\
Inositol & + & & a-arabinosidase \\
Lactose & + & & Motility \\
\hline
\end{tabular}


distress makes prey animals more vulnerable to predation [7]. While overt sickness behaviors occur with moribund animals, lack of expression of distress due to infection could lead to the potentially erroneous conclusion that post-surgical infection is extremely rare [31]. In an attempt to quantify the potentially confounding effects unapparent post-surgical infection could have on subsequent experiments, Bradfield et al. introduced bacteria following routine survival surgeries to induce post-surgical infections. Despite the absence of classic signs of distress, infection in rats significantly altered behavioral testing (open field apparatus) including exploratory and freezing behavior [32]. The volume and severity of inflammatory exudate in our case report subject indicates a systemic inflammatory response was ongoing despite an absence of distress and sickness behavior. Post-surgical abscesses within the abdomen do not necessarily cause the visible swelling that was present in our subject animal. Given that OVX is a common procedure in the in vivo study of osteoporosis and neurodegenerative diseases, conditions that would undoubtedly be affected by elevated inflammatory responses, post-surgical abscess is an important consideration to avoid confounding factors in rodent studies. While routine screening of blood for inflammatory markers of all subject animals in behavioral studies is likely impractical or costprohibitive, researchers employing behavioral testing may wish to consider collecting whole blood during necropsy and measuring white blood cell counts or inflammatory markers known to be elevated in the presence of abscess [32] in subjects displaying responses that substantially deviate from other members of their cohort.

\section{Abbreviation}

OVX: Ovariectomy

\section{Acknowledgements}

We wish to thank Peter Caradonna (UNE Histology and Imaging Core) for assistance with microscopy and Dr. Alexa Wakely (UNE Center for Excellence in the Neurosciences) for procedural training.

\section{Authors' contributions}

Surgical procedures/animal care: VEE, AE, TEK; postmortem evaluation, bacteriological analysis, bacterial identification: MM, SP; novel species characterization: SP, MM; study design and supervision: TEK, MM; manuscript composition/revision: VEE, TEK, KLH, MM; funding acquisition: TEK, KLH. All authors have read and approved this manuscript.

\section{Funding}

The study leading to this case report was funded by an intramural grant from the University of New England. The funding body had no role in the study design.

\section{Availability of data and materials}

Ribosomal RNA sequences have been deposited into GenBank, and are available under accession numbers MK167981, MK168019, and MK168057. Axenic cultures of bacterial isolates are available upon request with appropriate documentation to receive bacterial cultures.

\section{Ethics approval}

The study leading to this case report was approved by the University of New England's Institutional Animal Care and Use Committee (protocol \#052818-002).
Consent for publication

Not applicable.

\section{Competing interests}

The authors declare they have no competing interests.

\section{Author details}

${ }^{1}$ Department of Biomedical Sciences, University of New England, 11 Hills Beach Road, Biddeford, ME 04005, USA. ${ }^{2}$ Center of Excellences in the Neurosciences, University of New England, 11 Hills Beach Road, Biddeford, ME 04005, USA.

Received: 19 November 2018 Accepted: 3 October 2019

Published online: 24 October 2019

\section{References}

1. Arend P. Early ovariectomy reveals the germline encoding of natural anti-Aand Tn-cross-reactive immunoglobulin M (lgM) arising from developmental $\mathrm{O}-\mathrm{GalNAC}$ glycosylations. (Germline-encoded natural anti-a/Tn cross-reactive IgM). Cancer Med. 2017;6(7):1601-13.

2. Egermann M, Goldhahn J, Schneider E. Animal models for fracture treatment in osteoporosis. Osteoporos Int. 2005;16(Suppl 2):S129-38.

3. Ito M, Nishida A, Nakamura T, Uetani M, Hayashi K. Differences of threedimensional trabecular microstructure in osteopenic rat models caused by ovariectomy and neurectomy. Bone. 2002;30(4):594-8.

4. Labandeira-Garcia JL, Rodriguez-Perez Al, Valenzuela R, Costa-Besada MA, Guerra MJ. Menopause and Parkinson's disease. Interaction between estrogens and brain renin-angiotensin system in dopaminergic degeneration. Front Neuroendocrinol. 2016;43:44-59.

5. Burkholder T, Foltz C, Karlsson E, Linton CG, Smith JM. Health evaluation of experimental laboratory mice. Curr Protoc Mouse Biol. 2012;2:145-65.

6. Donnelly TM. Mice and Rats as Pets. In: Merck veterinary manual [internet]. Kenilworth: Merck \& CO, Inc.; 2018.

7. Mogil JS. Animal models of pain: progress and challenges. Nat Rev Neurosci. 2009;10(4):283-94.

8. Motyl KJ, Beauchemin M, Barlow D, Le PT, Nagano K, Treyball A, et al. A novel role for dopamine signaling in the pathogenesis of bone loss from the atypical antipsychotic drug risperidone in female mice. Bone. 2017;103: 168-76.

9. Montgomery C. Oncologic and toxicologic research: alleviation and control of pain and distress in laboratory animals. Cancer Bulletin. 1990:42:230-7.

10. May M, Brown DR. Mycoplasmas and related organisms. In: Goldman ER, green LH, editors. The practical handbook of microbiology. 3rd ed. Boca Raton: CRC Press; 2015.

11. Srivastava $S$, Singh V, Kumar V, Verma PC, Srivastava R, Basu V, et al. Identification of regulatory elements in 165 rRNA gene of Acinetobacter species isolated from water sample. Bioinformation. 2008;3(4):173-6.

12. Altschul SF, Madden TL, Schäffer AA, Zhang J, Zhang Z, Miller W, et al. Gapped BLAST and PSI-BLAST: a new generation of protein database search programs. Nucleic Acids Res. 1997;25(17):3389-402.

13. Stackebrandt E, Goebel BM. Taxonomic note: a place for DNA-DNA Reassociation and 165 rRNA sequence analysis in the present species definition in bacteriology. Int J Syst and Evol Microbiol. 1994:44:846-9.

14. Kumar S, Stecher G, Tamura K. MEGA7: molecular evolutionary genetics analysis version 7.0 for bigger datasets. Mol Biol Evol. 2016;33(7):1870-4.

15. Benedetti P, Rassu M, Branscombe M, Sefton A, Pellizzer G. Gemella morbillorum: an underestimated aetiology of central nervous system infection? J Med Microbiol. 2009;58(Pt 12):1652-6.

16. Milnik A, Gazis A, Tammer I, Bartels C. Immunocompetent young man with cerebral abscess and cortical venous infarction mimicking cerebritis caused by Gemella morbillorum. BMJ Case Rep. 2013;2013. https://doi.org/10.1136/ bcr-2012-007252.

17. Dewhirst FE, Klein EA, Thompson EC, Blanton JM, Chen T, Milella L, et al. The canine oral microbiome. PLoS One. 2012;7(4):e36067.

18. Leser TD, Amenuvor JZ, Jensen TK, Lindecrona RH, Boye M, Møller K. Culture-independent analysis of gut bacteria: the pig gastrointestinal tract microbiota revisited. Appl Environ Microbiol. 2002;68(2):673-90.

19. Li E, Hamm CM, Gulati AS, Sartor RB, Chen H, Wu X, et al. Inflammatory bowel diseases phenotype, C. difficile and NOD2 genotype are associated with shifts in human ileum associated microbial composition. PLoS One. 2012;7(6):e26284. 
20. Suchodolski JS, Camacho J, Steiner JM. Analysis of bacterial diversity in the canine duodenum, jejunum, ileum, and colon by comparative 16S rRNA gene analysis. FEMS Microbiol Ecol. 2008;66(3):567-78.

21. Borro P, Sumberaz A, Testino G. Pyogenic liver abscess caused by Gemella morbillorum. Colomb Med (Cali). 2014;45(2):81-4.

22. Chinbo M, Addebbous A, Moutachakkir M, Rada N, Bouskraoui M, Chabaa L, et al. Gemella haemolysans brain abscess in a child with complex congenital heart disease. Ann Biol Clin (Paris). 2014;72(4):487-90.

23. Chotai S, Moon HJ, Kim JH, Chung HS, Park YK, Kwon TH. Brain abscess caused by Gemella morbillorum: case report and review of the literature. Turk Neurosurg. 2012;22(3):374-7.

24. Hikone M, Sakamoto N, Ota M, Washino T, Kobayashi Kl, Iwabuchi S, et al. The first case report of infective endocarditis caused by Gemella taiwanensis. J Infect Chemother. 2017;23(8):567-71.

25. Mosquera JD, Zabalza M, Lantero M, Blanco JR. Endocarditis due to Gemella haemolysans in a patient with hemochromatosis. Clin Microbiol Infect. 2000; 6(10):566-8.

26. Zaidi SJ, Husayni T, Collins MA. Gemella bergeri infective endocarditis: a case report and brief review of literature. Cardiol Young. 2018;28(5):762-4.

27. Collins MD, Falsen E. Gemella. In: Bergey's Manual of Systematics of Archeae and Bacteria. Hoboken: Wiley; 2015.

28. Scharschmidt TC, List K, Grice EA, Szabo R, Renaud G, Lee CC, et al. Matriptase-deficient mice exhibit ichthyotic skin with a selective shift in skin microbiota. J Invest Dermatol. 2009;129(10):2435-42.

29. Lagkouvardos I, Pukall R, Abt B, Foesel BU, Meier-Kolthoff JP, Kumar N, et al. The mouse intestinal bacterial collection ( $\mathrm{miBC}$ ) provides host-specific insight into cultured diversity and functional potential of the gut microbiota. Nat Microbiol. 2016;1(10):16131.

30. Kodjo A, Villard L, Veillet F, Escande F, Borges E, Maurin F, et al. Identification by 165 rDNA fragment amplification and determination of genetic diversity by random amplified polymorphic DNA analysis of Pasteurella pneumotropica isolated from laboratory rodents. Lab Anim Sci. 1999;49(1):49-53.

31. Baker DG. Natural pathogens of laboratory mice, rats, and rabbits and their effects on research. Clin Microbiol Rev. 1998;11(2):231-66.

32. Bradfield JF, Schachtman TR, McLaughlin RM, Steffen EK. Behavioral and physiologic effects of inapparent wound infection in rats. Lab Anim Sci. 1992;42(6):572-8.

\section{Publisher's Note}

Springer Nature remains neutral with regard to jurisdictional claims in published maps and institutional affiliations.

Ready to submit your research? Choose BMC and benefit from:

- fast, convenient online submission

- thorough peer review by experienced researchers in your field

- rapid publication on acceptance

- support for research data, including large and complex data types

- gold Open Access which fosters wider collaboration and increased citations

- maximum visibility for your research: over $100 \mathrm{M}$ website views per year

At $\mathrm{BMC}$, research is always in progress.

Learn more biomedcentral.com/submissions 